\title{
Compiling Language Models from a Linguistically Motivated Unification Grammar
}

\author{
Manny Rayner ${ }^{\dagger \neq *}$, Beth Ann Hockey ${ }^{\dagger}$, Frankie James ${ }^{\dagger}$ \\ Elizabeth Owen Bratt ${ }^{\ddagger}$, Sharon Goldwater ${ }^{\ddagger}$ and Jean Mark Gawron ${ }^{\ddagger}$ \\ ${ }^{\dagger}$ Research Institute for \\ Advanced Computer Science \\ Mail Stop 19-39 \\ NASA Ames Research Conter \\ Moffett Field, CA 94035-1000 \\ ‡SRI International \\ 333 Ravenswood Ave \\ Menlo Park, CA 94025 \\ ${ }^{*}$ nctdecisions \\ Wellington House \\ East Road \\ Cambridge CB1 1BH \\ England
}

\begin{abstract}
Systems now exist which are able to compile unification grammars into language models that can be included in a speech recognizer, but it is so far unclear whether non-trivial linguistically principled grammars can be used for this purpose. We describe a series of experiments which investigate the question empirically, by incremontally constructing a grammar and discovering what problems emerge when successively larger versions are compiled into finite state graph representations and used as language models for a medium-vocabulary recognition task.
\end{abstract}

\section{Introduction $^{1}$}

Construction of speech recognizers for mediumvocabulary dialogue tasks has now become an important practical problem. The central task is usually building a suitable language model, and a number of standard methodologies have become established. Broadly speaking, these fall into two main classes. One approach is to obtain or create a domain corpus, and from it induce a statistical language model, usually some kind of $\mathrm{N}$-gram grammar; the alternative is to manually design a grammar which specifies the utterances the recognizer will accept. There are many theoretical reasons to prefer the first course if it is feasible, but in practice there is often no choice. Unless a substantial domain corpus is available, the only method that stands a chance of working is hand-construction of an ex-

\footnotetext{
'The majority of the research reported was performed at RIACS under NASA Cooperative Agrecment Number NCC 2-1006. The rescarch described in Section 3 was supported by the Defense Advanced Rescarch Projects Agency under Contract N66001-94-C-6046 with the Naval Command, Control, and Ocean Surveillance Center.
}

plicit grammar based on the grammar-writcr's intuitions.

If the application is simple enough, experience shows that good grammars of this kind can be constructed quickly and efficiently using commercially available products like ViaVoice SDK (IBM 1999) or the Nuance Toolkit ( $\mathrm{Nu}_{-}$ ance 1999). Systems of this kind typically allow specification of some restricted subset of the class of context-free grammars, together with annotations that permit the grammar-writer to associate semantic values with lexical entries and rules. This kind of framework is fully adequate for small grammars. As the grammars increase in size, however, the limited expressive power of context-free language notation becomes increasingly burdensome. The grammar tends to become large and unwieldy, with many rules appearing in multiple versions that constantly need to be kept in step with each other. It represents a large development cost, is hard to maintain, and does not usually port well to new applications.

It is tempting to consider the option of moving towards a more expressive grammar formalism, like unification grammar, writing the original grammar in unification grammar form and compiling it down to the context-free notation required by the underlying toolkit. At least one such system (Gemini; (Moore et al 1997)) has been implemented and used to build successful and non-trivial applications, most notably CommandTalk (Stent et al 1999). Gemini accepts a slightly constrained version of the unification grammar formalism originally used in the Core Language Engine (Alshawi 1992), and compiles it into context-free grammars in the GSL formalism supported by the Nuance Toolkit. The Nuance Toolkit compiles GSL grammars into sets of probabilistic finite state 
graphs (PFSGs), which form the final language model.

The relative success of the Gemini system suggests a new question. Unification grammars have been used many times to build substantial general grammars for English and other natural languages, but the language model oriented grammars so far devoloped for Gemini (including the one for CommandTalk) have all been domain-specific. One naturally wonders how feasible it is to take yet another step in the direction of increased generality; roughly, what wo want to do is start with a completely gencral, linguistically motivated grammar, combine it with a domain-specific lexicon, and compile the result down to a domain-specific contextfree grammar that can be used as a language model. If this programme can be realized, it is easy to believe that the result would be an extremely useful methodology for rapid construction of language models. It is important to note that there are no obvious theoretical obstacles in our way. The claim that English is contextfree has been respectable since at least the early 80s (Pullum and Gazdar 1982)2, and the idea of using unification grammar as a compact way of representing an underlying context-frec language is one of the main motivations for GPSG (Gazdar et al 1985) and other formalisms based on it. The real question is whether the goal is practically achicvable, given the resource limitations of current technology.

In this paper, we describe work aimed at the target outlined above, in which we used the Gemini system (described in more detail in Section 2) to attempt to compile a variety of linguistically principled unification grammars into language models. Our first experiments (Section 3) were performed on a large pre-existing unification grammar. These were unsuccessful, for reasons that were not entirely obvious; in order to investigate the problem more systematically, we then conducted a second series of experiments (Section 4), in which we incrementally built up a smaller grammar. By monitoring the beliavior of the compilation process and the resulting language model as the grammar's

\footnotetext{
${ }^{2}$ We are aware that this claim is most probably not true for natural languages in gencral (Bresnan ot al 1987), but further discussion of this point is beyond the scope of the paper.
}

coverage was expanded, we were able to identify the point at which serious problems began to emerge (Section 5). In the final section, we summarize and suggest further directions.

\section{The Gemini Language Model Compiler}

To make the paper more self-contained, this section provides some background on the method used by Gemini to compile unification grammars into CFGs, and then into language models. The basic idea is the obvious one: entumerate all possible instantiations of the features in the grammar rules and lexicon entries, and thus transform each rule and entry in the original unification grammar into a set of rules in the derived CFG. For this to be possible, the relevant features must bo constrained so that they can only take values in a finite predefined range. The finite range restriction is inconvenient for features used to build semantic representations, and the formalism consequently distinguishes syntactic and semantic foatures; semantic features are discarded at the start of the compilation process.

A naive implementation of the basic method world be impractical for any but the smallest and simplest grammars, and considerable ingenuity has been expended on various opti111\%ations. Most importantly, catcgories are expanded in a demand-driven fashion, with information being percolated both botton-up (from the lexicon) and top-down (from the grammar's start symbol). This is done in such a way that potentially valid combinations of feature instantiations in rules are successively filtered out if they are not licensed by the top-down and bottom-up constraints. Ranges of feature values are also kept together when possible, so that sets of context-free rules produced by the naive algorithm may in these cases be merged into single rules.

By exploiting the structure of the grammar and lexicon, the demand-driven expansion method can often effect substantial reductions in the size of the derived CFG. (For the type of grammar we consider in this paper, the reduction is typically by a factor of over $10^{20}$ ). The downside is that even an apparently small change in the syntactic features associated with a. rule may have a large effect on the size of 
the CFG, if it opens up or blocks an important percolation path. Addling or deleting lexicon entries can also have a significant effect on the size of the CFG, especially when there are only a small number of entries in a given grammatical category; as usual, entries of this type behave from a software engineering standpoint like grammar rules.

The language model compiler also performs a number of other non-trivial transformations. The most important of these is related to the fact that Nuance GSL grammars are not allowed to contain left-recursive rules, and leftrecursive unification-grammar rules must consequently be converted into a non-left-recursive form. Rules of this type do not however occur in the grammars described below, and we consequently omit further description of the method.

\section{Initial Experiments}

Our initial experiments wore performed on a recent unification grammar in the ATIS (Air Travel Information System) domain, developed as a linguistically principled grammar with a domain-specific lexicon. This grammar was created for an experiment comparing coverage and recognition performance of a handwritten grammar with that of automatically derived recognition language models, as increasing amounts of clata from the ATIS corpus were made available for each method. Examples of sentences covered by this grammar are "yes", "on friday", "i want to fly from boston to denver on united airlines on friday september twenty third", "is the cheapest one way fare from boston to denver a morning flight", and "what flight leaves earliest from boston to san francisco with the longest layover in denver". Problems obtaining a working recognition grammar from the unification grammar ended our original experiment prematurely, and led us to investigate the factors responsible for the poor recognition performance.

We explored several likely causes of recognition trouble: number of rules, number of vocabulary items, size of node array, perplexity, and complexity of the grammar, measured by average and highest number of transitions per graph in the PFSG form of the grammar.

We were able to immediately rule out simple size metrics as the cause of Nuance's diffi- culties with recognition. Our smallest air travel grammar had 14.1 Gemini rulos and 1043 words, producing a Nuance grammar with 368 rules. This compares to the CommandTalk grammar, which had 1231 Gemini rules and 1771 words, producing a Nuance grammar with 4096 rules.

To detcrmine whether the number of the words in the grammar or the structure of the phrases was responsible for the recognition problems, we created extreme cases of a Word+ grammar (i.e. a grammar that constrains the input to be any sequence of the words in the vocabulary) and a one-word-per-category grammar. We found that both of these variants of our grammar produced reasonable recognition, though the Word + grammar was very inaccurate. However, a thrce-words-per-category grammar could not produce successful speech recognition.

Many feature specifications can make a grammar more accurate, but will also result in a larger recognition grammar due to multiplication of feature values to derive the categories of the context-free grammar. We experimented with various techniques of selecting features to be retained in the recognition grammar. As doscribed in the previous section, Gemini's default method is to select only syntactic features and not consider semantic features in the recognition grammar. We experimented with selecting a subset of syntactic features to apply and with applying only scmantic sortal features, and no syntactic features. None of these grammars produced successful speech recognition.

¿From these experiments, we were unable to isolate any simple set of factors to explain which grammars would be problematic for speech recognition. However, the numbers of transitions per graph in a PFSG did seem suggestive of a factor. The ATIS grammar had a high of 1184 transitions per graph, while the semantic grammar of CommandTalk had a high of 428 transitions per graph, and produced very reasonable speech recognition.

Still, at the end of these attempts, it became clear that we did not yet know the precise characteristic that makes a linguistically motivated grammar intractable for speech recognition, nor the best way to retain the advantages of the hand-written grammar approach while providing reasonable speech recognition. 


\section{Incremental Grammar Development}

In our second series of experiments, we incrementally developed a now grammar from scratch. The now grammar is basically a scaleddown and alapted version of the Core Language Engine grammar for English (Pulman 1992; Rayner 1993); concrete development work and testing were organized around a speech interface to a set of functionalities offered by a simple simulation of the Space Shuttle (Rayner, Hockey and James 2000). Rules and lexical entries were added in small groups, typically $2-3$ rules or 5-10 lexical entries in one incremont. After each round of expansion, wo tested to make sure that the grammar could still be compiled into a usable recognizer, and at sevcral points this suggested changes in our inplementation strategy. The rest of this section describes the new grammar in more detail.

\subsection{Overview of Rules}

The current versions of the grammar and lexicon contain 58 rules and $30 j$ uninflected entrios respectively. They cover the following phenomclla:

1. Top-level ntterances: declarative clanses, WH-questions, Y-N questions, imperatives, clliptical NPs and PPs, interjections.

2. WH-movement of NPs and PPs.

3. The following verb types: intransitive, simple transitive, PP complement, modal/auxiliary, -ing VP complement, particle+NP complement, sentential complement, embedded question complement.

4. PPs: simple PP, PP with postposition ("ago"), PP modification of VP and NP.

5. Rolative clauses with both relative NP pronoun ("the temperature that I measured") and relative PP ("the deck where I am").

6. Numeric determiners, time expressions, and postmodification of NP by numeric expressions.

7. Constituent conjunction of NPs and clauses.

The following example sentences illustrate current coverage: "yes", "how about scenario three?", "what is the temperature?", "measure the pressure at flight deck", "go to the crew latel and close it", "what were temperature and pressure at fifteon oh five?", "is the temperature going up?", "do the fixed sensors say that the pressure is decreasing?", "find out when the pressure reached fifteen ps i", "what is the pressure that you measured?", "what is the temperature where you are?", "can you find out when the fixed sensors say the temperature at flight deck reached thirty degrees celsius?".

\subsection{Unusual Features of the Grammar}

Most of the grammar, as already stated, is closely based on the Core Language Engine grammar. We briefly summarize the main divergences between the two grammars.

\subsubsection{Inversion}

The new grammar uses a novel treatment of inversion, which is partly designed to simplify the process of compiling a feature grammar into context-free form. The CLE grammar's treatment of inversion uses a movement account, in which the fronted verb is moved to its notional place in the VP through a feature. So, for example, the sentence "is pressure low?" will in the original CLE grammar have the phrasestruct,ure

\section{"[[is $\left.\left.]_{V}[\text { pressurc }]_{N I},\left[[]_{V}[\mathrm{low}]_{A D J}\right]_{V P}\right]\right]_{S} "$}

in which the head of the VP is a $V$ gap coindexed with the fronted main verb "is".

Our new grammar, in contrast, handles inversion without movement, by making the combination of inverted verb and subject into a VBAR constitucnt. A binary feature invsubj picks out these VBARs, and there is a questionformation rule of the form

$$
S \rightarrow V P:[\text { invsubj=y] }
$$

Continuing the example, the new grammar assigns this sentence the simpler phrasestructure

$$
\text { "[[[is } \left.\left.\left.]_{V}[\text { pressure }]_{N P}\right]_{V B A R}\left[[\mathrm{low}]_{A D J}\right]_{V P}\right]\right]_{S}
$$

\subsubsection{Sortal Constraints}

Sortal constraints are coded into most grammar rules as syntactic features in a straight-forward manner, so they are available to the compilation 
process which constructs the context-free grammar, and ultimately the language model. The current lexicon allows 11 possible sortal values for nouns, and 5 for PPs.

We have taken the rather non-standard step of organizing the rules for PP modification so that a VP or NP cannot be modified by two PPs of the same sortal type. The principal motivation is to tighten the language model with regard to prepositions, which tend to be phonetically reduced and often hard to distinguish from other function words. For example, without this extra constraint we discovered that an utterance like

measure temperature at flight deck and lower deck

would frequently be misrecognized as

measure temperature at flight deck in lower deck

\section{Experiments with Incremental Grammars}

Our intention when developing the new grammar was to find out just when problems began to emerge with respect to compilation of language models. Our initial hypothesis was that these would probably become serious if the rules for clansal structure were reasonably elaborate; wo expected that the large number of possible ways of combining modal and auxiliary verbs, question formation, movement, and sentential complements would rapidly combine to produce an intractably loose language model. Interestingly, this did not prove to be the case. Instead, the rules which appear to be the primary cause of difficulties are those relating to relative clanses. We describe the main results in Section 5.1; quantitative results on recognizer performance are presented together in Section 5.2.

\subsection{Main Findings}

We discovered that addition of the single rule which allowed relative clause modification of an NP had a drastic effect on recognizer performance. The most obvious symptoms were that recognition became much slower and the size of the recognition process much larger, sometimes causing it to exceed resource bounds. The false reject rate (the proportion of uttcrances which fell below the recognizer's minimum confidence theshold) also increased substantially, though we were surprised to discover no significant increase in the word error rate for sentences which did produce a recognition result. To investigate the cause of these effects, we examined the results of performing compilation to GSL and PFSG level. The compilation processes are such that symbols retain mnemonic names, so that it is relatively easy to find GSL rules and graphs used to recognize phrases of specified grammatical categories.

At the GSL level, addition of the relative clause rule to the original unification grammar only increased the number of derived Nuance rules by about $15 \%$, from 4317 to 4959 . The average size of the rules however increased much more $^{3}$. It is casiest to measure sizc at the level of PFSGs, by counting nodes and transitions; we found that the total size of all the graphs had increased from 48836 nodes and 57195 transitions to 113166 nodes and 140640 transitions, rather more than doubling. The increase was not distributed cvenly between graphs. We extracted figures for only the graphs relating to specific grammatical categories; this showed that the number of graphs for NPs had increased from 94 to 258 , and morcover that the average size of each NP graph had increased from 21 nodes and 25.5 transitions to 127 nodes and 165 transitions, a more than sixfold increase. The graphs for clause (S) phrases had only increased in number from 53 to 68 . They had however also greatly increased in average size, from 171 nodes and 212 transitions to 445 nodes and 572 transitions, or slightly less than a threefold increase. Since NP and $S$ are by far the most important; categories in the grammar, it is not strange that these large changes make a great difference to the quality of the language model, and indirectly to that of speech recognition.

Comparing the original unification grammar and the compiled GSL version, we were able to make a precise diagnosis. The problem with the relative clause rules are that they unify feature values in the critical $S$ and NP subgrammars; this means that each constrains the other, leading to the large observed increase in the size and complexity of the derived Nuance grammar.

\footnotetext{
${ }^{3}$ GSL rules are written in an notation which allows disjunction and KKlcenc star.
} 
Specifically, agreement information and sortal category are shared between the two danghter NPs in the relative clause modification rule, which is schematically as follows:

$$
\begin{aligned}
& \text { NP }:[\operatorname{agr}=A, \text { sort }=\mathrm{S}] \\
& \text { NP: }[\operatorname{agr}=\mathrm{A}, \text { sort }=\mathrm{S}] \\
& \text { REL }:[\operatorname{agr}=\mathrm{A}, \text { sort }=\mathrm{S}]
\end{aligned}
$$

These feature settings are needed in order to get the right alternation in pairs like

the robot that *measure/measures the temperature [agr]

the *deck/temperature that you measured [sort]

We tested our hypothesis by commenting out the agr and sort features in the above rule. This completely solves the main problem of explosion in the sire of the PFSG representation; the new version is only very slightly larger than the one with no relative clatise rule (50647 nodes and 59322 transitions against 48836 nodes and 57195 transitions). Most importantly, there is no great increase in the number or average sizo of the NP and S graphs. NP graplss increase in number from 94 to 130 , and stay constant in average size; $S$ graphs increase in number from 53 to 64 , and actually decrease in average size to 135 nodes and 167 transitions. Tests on speech data show that recognition quality is nearly the sane as for the version of the recognizer which does not cover relative clanses. Although speed is still significantly degraded, the process size has been reduced sufficiently that the problems with resource bounds disappear.

It would be reasonable to expect that removing the explosion in the PFSG representation would result in an underconstiained language model for the relative clause part of the grammar, causing degraded performance on utterances containing a relative clause. Interestingly, this does not appear to happen, though recognition speed under the new grammar is significantly worse for these utterances compared to utterances with no relative clause.

\subsection{Recognition Results}

This section summarizes our empirical recognition results. With the help of the Nuance Toolkit batchrec tool, we evaluated three versions of the recognizer, which differed only with respect to the language model. no.rels used the version of the language model derived from a grammar with the relative clause rule removed; rels is the version derived from the full grammar; and unlinked is the compromise version, which kecps the relative clause rule but removes the critical features. We constructed a corpus of 41 utterances, of mean length 12.1 words. The atterances were chosen so that the first 31 were within the coverage of all three versions of the grammar; the last 10 contained relative clauses, and were within the coverage of rels and unlinked but not of no_rels. Each utterance was recorded by eight different subjects, none of whom had participated in development; of the grammar or recognizers. Tests were rum on a dial-processor SUN Ultra60 with 1.5 GB of RAM.

The recogniyer was set to reject utterances if their associated confidence measure fell under the default threshold. Figures 1 and 2 summarime the results for the first 31 utterances (no relative clauses) and the last 10 utterances (rclative clauses) respectively. Under 'xRT', we give mean recognition speed (averaged over subjects) expressed as a multiple of real time; 'FRej' gives the falso reject rate, the mean percentage of utterances which were rejected due to low confidence measures; 'Mem' gives the mean percentage of utterances which failed due to the recognition process exceding memory resource bounds; and 'WER' gives the mean word crror rate on the sentences that were neither rejected nor failed due to resource bound problems. Since the distribution was highly skewerl, all means were calculated over the six subjects remaining after exclusion of the extreme ligh and low values.

Looking first at Figure 1, we sce that rels is clearly inferior to no_rels on the subset of the corpus which is within the coverage of both versions: nearly twice as many utterances are rejected due to low confidence values or resource problems, and recognition speed is about five times slower. unlinked is in contrast not significantly worse than no_rels in terms of recognition performance, though it is still two and a half times slower.

Figure 2 compares rels and unlinked on the utterances containing a relative clause. It scems reasonable to say that recognition performance 


\begin{tabular}{|c|r|r|r|r|}
\hline Grammar & xRT & FRej & Mem & WER \\
\hline \hline no_rels & 1.04 & $9.0 \%$ & - & $6.0 \%$ \\
\hline rels & 4.76 & $16.1 \%$ & $1.1 \%$ & $5.7 \%$ \\
\hline unlinked & 2.60 & $9.6 \%$ & - & $6.5 \%$ \\
\hline
\end{tabular}

Figure 1: Evaluation results for 31 utterances not containing relative clauses, averaged across 8 subjects excluding extreme values.

\begin{tabular}{|c|c|c|c|c|}
\hline Grammar & xRT & FRej & Mem & WER \\
\hline \hline rels & 4.60 & $26.7 \%$ & $1.6 \%$ & $3.5 \%$ \\
\hline unl.inked & 5.29 & $20.0 \%$ & - & $5.4 \%$ \\
\hline
\end{tabular}

Figure 2: Evaluation results for 10 utterances containing relative clauses, averaged across 8 subjects excluding extreme values.

is comparable for the two versions: rels has lower word error rate, but also rejects more utterances. Recognition speed is marginally lower for unlinked, though it is not clear to us whether the difference is significant given the high variability of the data.

\section{Conclusions and Further Directions ,}

We found the results presented above surprising and interesting. When we began our programme of attempting to compile increasingly larger linguistically based unification grammars into language models, we had expected to see a steady combinatorial increase, which we guessed would be most obviously related to complex clause structure. This did not turn out to be the case. Instead, the serious problems we encountered were caused by a small number of critical rules, of which the one for relative clause modification was by the far the worst. It was not immediately obvious how to deal with the problem, but a careful analysis revealed a reasonable compromise solution, whose only drawback was a significant but undisastrous degradation in recognition speed.

It seems optimistic to hope that the relative clause problem is the end of the story; the obvious way to investigate is by continuing to expand the grammar in the same incremental fashion, and find out what happens next. We intend to do this over the next few months, and expect in due course to be able to present further: results.

\section{References}

H. Alshawi. 1992. The Core Language Engine. Cambridge, Massachusetts: The MIT Press.

J. Bresnan, R.M. Kaplan, S. Peters and A. Za-enen. Cross-Serial Dependencies in Dutch. 1987. In W. J. Savitch et al (eds.), The Formal Complexity of Natural Language, Reidel, Dordrecht, pages 286-319.

G. Gazdar, E. Klein, G. Pullum and I. Sag. 1985. Generalized Phrase Structure Grammar Basil Blackwell.

IBM. 1999. ViaVoice SDK for Windows, version 1.5.

R. Moore, J. Dowding, H. Bratt, J.M. Gawron, Y. Gorfu, and A. Cheyer. 1997. CommandTalk: A Spoken-Language Interface for Battleficld Simulations. Proceedings of the Fifth Conference on Applied Natural Language Processing, pages $1-7$, Washington, DC. Available online from http://ww.ai.sri.com/natural-language /projects/arpa-sls/commandtalk.html.

Nuance Communications. 1999. Nuance Speech Recognition System Developer's Manual, Version 6.2

G. Pullum and G. Gazdar. 1982. Natural Languages and Context-Free Languages. Linguistics and Philosophy, 4, pages 471-504.

S.G. Pulman. 1992. Unification-Based Syntactic Analysis. In (Alshawi 1992)

M. Rayner. 1993. English Linguistic Coverage. In M.S. Agnäs et al. 1993. Spoken Language Translator: First Year Report. SRI Technical Report CRC-043. Available online from http://www.sri.com.

M. Rayner, B.A. Hockcy and F. James. 2000. Turning Speech into Scripts. To appear in Proceedings of the 2000 AAAI Spring Symposium on Natural Language Dialogues with Practical Robotic Devices

A. Stent, J. Dowding, J.M. Gawron, E.O. Bratt, and R. Moore. 1999. The CommandTalk Spoken Dialogue System. Proceedings of the 37th Annual Meeting of the $A C L$, pages 183-190. Available online from http://ww .ai.sri.com/natural-language /projects/arpa-sls/commandtalk.html. 